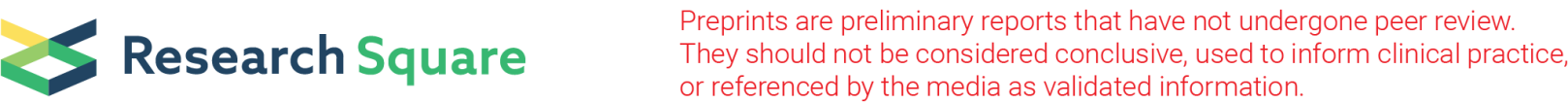

\section{Dearomative [4 + 3] cycloaddition of furans with vinyl- $N$-triftosylhydrazones by silver catalysis: stereoselective access to oxa-bridged seven- membered bicycles}

\author{
Zhaohong Liu \\ Northeast Normal University \\ Yong Yang \\ Northeast Normal University \\ Xinyu Jiang \\ Northeast Normal University \\ Qingmin Song \\ Northeast Normal University \\ Giuseppe Zanoni \\ University of Pavia \\ Shaopeng Liu \\ Northeast Normal University \\ Xihe Bi ( $\sim$ bixh507@nenu.edu.cn ) \\ Northeast Normal University https://orcid.org/0000-0002-6694-6742
}

\section{Article}

Keywords:

Posted Date: January 4th, 2022

DOI: https://doi.org/10.21203/rs.3.rs-1195526/v1

License: (c) (i) This work is licensed under a Creative Commons Attribution 4.0 International License.

Read Full License

Version of Record: A version of this preprint was published at Organic Chemistry Frontiers on January 1st, 2022. See the published version at https://doi.org/10.1039/D2Q000256F. 


\section{Abstract}

The first example of dearomative [4 + 3] cycloaddition between furans and vinyl- $N$-sulfonylhydrazones as vinylcarbene precursors is reported. The merger of silver catalysis and easily decomposable vinyl- $\mathrm{N}$ triftosylhydrazones enabled the efficient synthesis of a variety of skeletally and functionally diverse oxabridged seven-membered bicyclic compounds with complete and predictable stereoselectivity. The combination of experimental studies and DFT calculations disclosed that the silver-catalyzed reaction proceeds via a concerted [ $4+3]$ cycloaddition mechanism, rather than the generally accepted cyclopropanation / Cope rearrangement pathway by rhodium catalysis.

\section{Introduction}

Seven-membered carbocycles, especially those embedded in oxa-bridged bicyclic scaffolds, are privileged structural motifs widely found in natural products and synthetic drugs. ${ }^{1-7}$ Over the past few decades, the [4 + 3] cycloaddition between 1,3-dienes and vinylcarbenes has been established as the most promising strategy for the stereoselective synthesis of these complex ring systems, as demonstrated by a variety of complex natural products synthesis. ${ }^{8-13}$ Major efforts in this arena so far have been dedicated to the use of vinyl diazo compounds, ${ }^{14-18}$ propargyl esters, ${ }^{19-21}$ 4-alkenyl-1,2,3-triazoles, ${ }^{22,23}$ enynes, ${ }^{24-}$

27 allenes, ${ }^{28,29}$ and 7-vinyl-1,3,5-cycloheptatrienes ${ }^{30}$ as the sources of vinylcarbene partners to access 1,4cycloheptadienes (Fig. 1a). However, the use of furans as cyclic 1,3-diene equivalents in such [4+3] cycloaddition reactions to construct oxa-bridged seven-membered bicyclic skeletons remained a challenging task, mainly due to the unfavorable entropic and transannular interaction. ${ }^{31}$ After the seminal contribution from the group of Davies in 1985, the few available examples in the literature are limited to the use of hazardous vinyl diazoacetates as donor-acceptor vinylcarbene precursors through a rhodiumcatalyzed cyclopropanation / Cope rearrangement sequence (Fig. 1b). While the rhodium-catalyzed protocol has been applied in the total syntheses of several natural products, ${ }^{36-39}$ this method is still problematic in terms of efficiency and practicality, including (i) delivering a mixture of cycloadduct and triene byproduct, which is highly dependent on the substitution pattern of furans, ${ }^{36,37}$ and (ii) unsuitable for alkyl- and unsubstituted vinyl diazo compounds. Moreover, the use of other vinylcarbene precursors such as cyclopropenes and propargylic carboxylates all exclusively resulted in trienes through the carbene-triggered ring-opening of furans. ${ }^{40-42}$ Therefore, a new catalytic approach to achieve the stereoselective synthesis of seven-membered oxa-bridged bicycles by the [4 +3$]$ cycloaddition of furans with vinylcarbenes is highly in demand.

As part of our continued efforts in the design and application of functionalized $N$-triftosylhydrazones, ${ }^{43-46}$ we were intrigued by the possibility of applying this approach to solve the aforementioned issues in the [4 +3 ] cycloaddition methodology. Here we report a silver-catalyzed dearomative [4 +3] cycloaddition of furans using the easily decomposable vinyl-N-triftosylhydrazones as donor and donor-acceptor vinylcarbene precursors (Fig.1c). The use of vinyl- $\mathrm{N}$-sulfonylhydrazones as vinylcarbene precursors is known to be challenging because they easily tend to form pyrazoles by self-cyclization. ${ }^{47,48} \mathrm{We}$ 
speculated that this could be addressed by the combined use of silver catalysis and vinyl- $N$ triftosylhydrazones. Moreover, mechanistic investigations reveal that the silver-catalyzed reaction proceeds through a concerted [4 +3] cycloaddition, rather than the cyclopropanation / Cope rearrangement sequence by rhodium catalysis, thus mechanically avoiding the formation of triene byproducts.

\section{Results}

The study began by determining the optimal conditions for the cycloaddition of vinyl- $N$-triftosylhydrazone 1a with furan using $\mathrm{NaH}$ as the base in the presence of varied catalyst and solvent (Table 1). The use of AgOTf as a catalyst in $\mathrm{CHCl}_{3}$ at $60^{\circ} \mathrm{C}$ ensued the desired product 2 in $24 \%$ yield, with a carbene dimer as the major product (Entry 1 ). The product yield could be substantially improved when using weakly

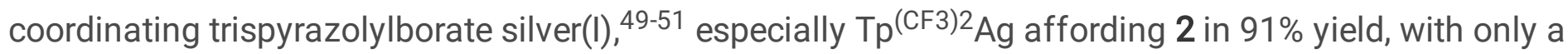
trace amount of undesired triene byproduct (entries 2 and 3). In contrast, $\mathrm{Rh}_{2}(\mathrm{OAc})_{4}{ }^{14}$ and $\mathrm{Cu}(\mathrm{hfacac})_{2}{ }^{18}$ the most commonly used catalysts in the [4 +3$]$ cycloaddition of 1,3-dienes with vinyldiazoacetates, led to low yield, whereas $\mathrm{Pd}(\mathrm{OAc})_{2}$ only enabled the carbene dimerization and the self-cyclization to pyrazole (entries 4-6). Further, the screening on solvents disclosed that $\mathrm{CH}_{2} \mathrm{Cl}_{2}$ and $\mathrm{PhCF}_{3}$ were less effective than $\mathrm{CHCl}_{3}$, whereas 1,4-dioxane was completely ineffective (entries 7-9). Vinyl- $\mathrm{N}$-tosylhydrazone $\mathbf{1 b}$ as vinylcarbene source, instead of the easily decomposable vinyl-N-triftosylhydrazone 1a, afforded $\mathbf{2}$ in a far low yield (40\%), owing to the self-cyclization to pyrazole (entry 10). The structural configuration of 2 was unambiguously established by the X-ray crystallographic analysis. ${ }^{52}$

With the optimized conditions in hand (Table 1, entry 3), the substrate scope of this reaction with a series of furans was then investigated. As shown in Fig. 2, furans with various substituents at 2-position, including alkyl, alkenyl, alkynyl, and (hetero)aryl as well as bromo, tributylstannyl, siloxy, allyl, and benzyl ether, ester, and ketone functional groups reacted smoothly with phenyl vinyl- $N$-triftosylhydrazone $\mathbf{3}$ to afford the corresponding products (4-18) in 44-90\% yield. We were pleased to find that the substrates bearing a carbon-carbon double/triple bond and an activated $\mathrm{C}\left(\mathrm{sp}^{3}\right)-\mathrm{H}$ bond (15-18) did not undergo competitive $[2+1]$ cycloaddition ${ }^{53-55}$ and $\mathrm{C}-\mathrm{H}$ insertion, ${ }^{10}$ thus achieving high chemoselectivity. The relatively low yields observed in some cases were ascribed to the ring-opening of furans to trienes and the carbene dimerization to olefins. In comparison, 2-substituted furans resulted in trienes as the major product in rhodium-catalyzed [4 +3] cycloaddition with alkenyl diazoacetates. ${ }^{[12 a-c]}$ In addition, the steric hindrance of furans had no apparent effect on the reaction, for instance, di- and tri-substituted furans reacted efficiently with 3 , providing multi-substituted oxa-bridged bicycles $19-23$ in 55-87\% yield.

Next, we investigated the reaction scope with respect to vinyl- $N$-triftosylhydrazones. As summarized in Fig. 2, a broad range of $\beta$-aryl vinyl- $N$-triftosylhydrazones with both electron-donating groups (e.g., Me and $\mathrm{OMe}$ ) and -withdrawing groups (e.g., halogen, $\mathrm{CN}_{1} \mathrm{NO}_{2}, \mathrm{CO}_{2} \mathrm{Et}$, and $\mathrm{CF}_{3}$ ) at various positions of aryl ring afforded the corresponding oxa-bridged bicyclic products (24-35) in 68-85\% yield. Similarly, vinyl- $N$ triftosylhydrazones bearing an indolyl, thienyl, or furyl group at $\beta$-position all efficiently reacted with furan 
to afford the heteroaryl-substituted products (36-38) in 72-81\% yield, whereas a ferrocenyl-substituted substrate led to the product (39) in a moderate yield (45\%). The tolerance of strongly coordinated heteroatoms renders this silver-based catalytic approach particularly useful for the construction of medicinally relevant heterocyclic molecules. Moreover, the reaction of $\mathrm{N}$-triftosylhydrazones derived from 2,4-dienals produced 4-vinyl oxa-bridged bicycles (40-42) in good yield, and the vinyl group present in the reaction products allows further orthogonal derivatization. In addition, an ester-containing product (43, $54 \%$ yield) was readily accessed from the carbethoxy-substituted vinyl- $N$-triftosylhydrazone. Alkylsubstituted vinyldiazomethanes are known to be prone to spontaneous cyclization to pyrazoles within hours even stored at $-20^{\circ} \mathrm{C} .{ }^{21,56}$ By using the strategy of the merge of silver catalysis and vinyl- $N$ triftosylhydrazones, we were pleased to find that $\beta$-alkyl vinyl- $N$-triftosylhydrazones smoothly reacted with furan to give the corresponding 4-alkyl-8-oxabicyclo[3.2.1] oct-2,6-dienes (44-49, 62-85\% yield), in which the common functional groups, including ester (OAc, 47), benzyloxy $(\mathrm{OBn}, 48)$, and dimethyltertbutylsilyloxy (OTBS, 49), were well tolerated. Furthermore, the substitution at a-position with an alkyl or halogen atom did not affect the efficiency and stereoselectivity (50-53, 67-84\% yield). $N$ triftosylhydrazones derived from a, $\beta$-unsaturated ketones also underwent this reaction, even though an electron-withdrawing substituent at carbene carbon is required to suppress the self-cyclization to pyrazoles (54-61, 53-94\% yield). ${ }^{2}$ Note that the compounds 60 and 61 could be prepared by rhodiumcatalyzed method, albeit slow addition of styryldiazoacetate was required to achieve good efficiency. ${ }^{35}$ Furthermore, unsubstituted allyl aldehyde-derived $N$-triftosylhydrazone was examined in the current protocol; to our delight, the desired oxa-bridged bicyclic product 62 was obtained in $72 \%$ yield. This achievement clearly demonstrated the superiority of current method, as unsubstituted vinyl carbenes underwent self-condensation more easily than the substituted ones. ${ }^{56,57}$ Overall, the results summarized in Fig. 2 clearly demonstrated the superior features of silver-catalyzed [ $4+3]$ cycloaddition between furans and vinyl- $N$-triftosylhydrazones, namely the broad substrate scope, good functional group tolerance, excellent chemo-, regio- and endo-stereoselectivity, and good to high yield, thereby constituting a versatile method for the stereoselective synthesis of a wide range of endo oxa-bridged 1,4cycloheptadienes.

Encouraged by the above achievements, we then turned our attention towards the synthesis of fused [5.n.0] bicyclic systems, a challenging target that has attracted the interest of synthetic and medicinal chemists due to their structural diversity and biological relevance (Fig. 3). ${ }^{1,58}$ First, we explored the [4 + 3] cycloaddition of furan with cyclic vinyl- $N$-triftosylhydrazones derived from cyclic enals, which were easily prepared by the one-carbon homologation reaction from commercially available cyclic ketones. ${ }^{59} \mathrm{~A}$ series of cyclic vinyl- $N$-triftosylhydrazones, having 6 to 8 membered rings, were found to be suitable in this reaction, affording the desired fused [5.4.0], [5.5.0], and [5.6.0] bicyclic compounds (63-66) in 53-78\% yield. Furthermore, several commercially available 2,3-fused cyclic furans were suitable for this [4+3] cycloaddition, delivering the corresponding oxa-bridged [5.4.0] bicyclic compounds (67-70) in 49-80\% yield. Finally, the intramolecular [4 + 3] cycloaddition of diverse vinyl-N-triftosylhydrazones bearing a tethered furan was rationally designed and applied to prepare benzannulated bicyclic compounds, which are the common structural core of several biologically active natural products. ${ }^{60}$ We were pleased to find 
that these intramolecular [4 +3] cycloaddition reactions proceeded smoothly under the silver-catalyzed conditions, affording the desired benzannulated bicyclic compounds (71-74) in 60-76\% yield. To the best of our knowledge, this is the first example of an intramolecular type I [4 +3] cycloaddition between vinylcarbenes and furans. ${ }^{61}$

To demonstrate the practicality of the protocol, a gram-scale reaction between hydrazone $\mathbf{7 5}$ and furan was performed under standard conditions, affording the product $\mathbf{5 0}$ in $80 \%$ yield $(1.05 \mathrm{~g})$ that is comparable to that obtained on small scale (Fig. 4). Further, the silver-catalyzed [4 +3 ] cycloaddition protocol was applied to the late-stage modification of drug molecules, such as Aspirin, Indomethacin, and Fenbufen bearing a furan motif. All these compounds reacted smoothly with vinyl- $N$-triftosylhydrazone 3 and gave the corresponding oxa-bridged [3.2.1] bicyclic compounds (76-78) in synthetically useful yield.

Based on related precedents, $8,13,30,32$ this transformation may proceed via a tandem cyclopropanation / Cope rearrangement sequence. Therefore, our initial mechanistic studies aimed to distinguish a direct [ $4+$ 3] cycloaddition pathway and a tandem [2 + 1] cycloaddition followed by a divinylcyclopropane [3,3]rearrangement. To address this, we first tried to capture the furanocyclopropane intermediate by shortening the reaction time or performing the reaction at a low temperature $\left(0{ }^{\circ} \mathrm{C}\right)$, but all failed (for details, see Fig. S1). Fortunately, the use of vinyl- $N$-triftosylhydrazone $\mathbf{7 9}$ in the reaction with furan produced a mixture of vinylcyclopropane $\mathbf{8 0}$ (which would correspond to the putative intermediates of the stepwise process) ${ }^{13,32}$ and triene $\mathbf{8 1}$, along with a trace amount of 1,4-cycloheptadiene $\mathbf{8 2}$. When the isolated compound 80 was heated at $160^{\circ} \mathrm{C}$ for 12 hours, only a triene 81 was isolated in $90 \%$ yield by the ring-opening of furanocyclopropane intermediate, whereas no rearrangement product $\mathbf{8 2}$ was observed (Fig. 5a). This result is different from the Rh-catalyzed [4 +3] cycloaddition of 1,3-dienes with vinylcarbenoids. ${ }^{13,30,32}$ Finally, we got a circumstantial evidence from the analysis of regioselectivity in the reaction of 3-phenylfuran with vinyl- $N$-triftosylhydrazone $\mathbf{3}$, which afforded a 1:1 mixture of $\mathbf{8 3}$ to $\mathbf{8 3}$ '. However, this is not consistent with the stereospecificity resulting from a tandem cyclopropanation / Cope rearrangement, where the initial cyclopropanation should selectively occur at the double bond with less sterical hindrance. ${ }^{13,30,32}$ Hence, the involvement of a concerted [ $\left.4+3\right]$ cycloaddition is more possible than a step-wise tandem cyclopropanation / Cope rearrangement.

To support our hypothesis concerning the involvement of a concerted [4 +3$]$ cycloaddition, in which silver vinylcarbenes act as a delocalized $2 \pi$ system, ${ }^{62}$ density functional theory (DFT) calculations were carried out for a model reaction, namely the silver-catalyzed reaction of 2-methylfuran with vinyl- $N$ triftosylhydrazone $\mathbf{3}$ (for computational details see Fig. S2-S5 in supporting information). First, the diazo

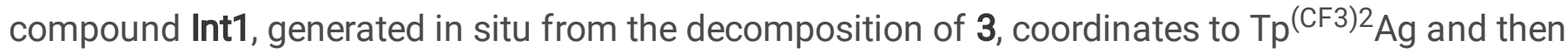
releases a $\mathrm{N}_{2}$ molecule to generate a silver carbene Int2 with an energy barrier of $11.3 \mathrm{kcal} / \mathrm{mol}$ (Fig. S3). Subsequently, a concerted [4+3] cycloaddition of Int2 with 2-methylfuran occurs affording 1,4cycloheptadiene products 4, 4', and 4", respectively, due to the approach of carbene intermediate Int2 to 2methylfuran from different orientations (Fig. 5b and Fig. S4). The energy barrier for the generation of the 
desired product 4 from Int3-B1 via TS2-4 is only $1.8 \mathrm{kcal} / \mathrm{mol}$, which is 12.0 and $23.4 \mathrm{kcal} / \mathrm{mol}$ lower than those for the formation of regioisomer 4' via TS2-4' from Int3-B2 and stereoisomer 4" via TS2-4" from Int3-C. To investigate the origin of the regio- and stereoselectivity, $\mathrm{NCl}$ and frontier molecular orbital analyses of transition states were performed (Fig. $5 \mathrm{C}$ and Fig. S2) ${ }^{63,64} \mathrm{~A}$ secondary orbital interaction exists in the boat form of transition states TS2-4 and TS2-4', and remarkably lowers the energy barriers for the generation of endo products and determines the stereoselectivity of this silver-catalyzed [4+3] cycloaddition. Furthermore, due to the $\mathrm{C}-\mathrm{H} \cdots \pi$ interactions between methyl and phenyl groups in TS2-4 and the steric exclusion between the methyl group and the bulky $\mathrm{Tp}^{(\mathrm{CF} 3)^{2}}$ ligand of carbene moiety in TS2-4' (Figure 2c, for details see Figure S2), the secondary orbital interaction in TS2-4' is weaker than that in TS2-4. This result provides a suitable rationale for high regioselectivity observed in the experiment. By comparison, the DFT calculations for the tandem cyclopropanation / Cope rearrangement pathway have been carried out, but this process entails an energy barrier $(14.1 \mathrm{kal} / \mathrm{mol})$ that is higher than that for concerted [4 +3] cycloaddition pathway $(1.8 \mathrm{kal} / \mathrm{mol})$ (see Fig. S5). At this moment, we cannot rule out completely that a tandem cyclopropanation / Cope rearrangement pathway is involved, but the experimental results and theoretical calculations indicate that a concerted [ $4+3]$ cycloaddition pathway is more favorable.

\section{Discussion}

In conclusion, we have developed a novel dearomative [4 +3] cycloaddition of furans with donor and donor-acceptor vinylcarbenes by the combination of silver catalysis and vinyl- $N$-triftosylhydrazones. This reaction features broad substrate scope, excellent functional group tolerance, complete and predictable chemo-, regio- and stereoselectivity, thus constituting constitute a powerful method for the synthesis of skeletally and functionally diverse oxa-bridged [3.2.1] bicyclic compounds from simple starting materials under mild conditions. Investigation of the mechanism by experimental studies and DFT calculations disclosed a concerted [4+3] cycloaddition pathway. Investigations into further extensions to other heteroaromatic molecules as well as into the development of an asymmetric version are ongoing in our laboratory.

\section{Declarations}

Data availability

Complete experimental procedures and compound characterization data are available in the Supplementary Information or from the corresponding author upon request

Acknowledgements

This work was supported by NSFC $(21871043,21961130376)$, Department of Science and Technology of Jilin Province (20180101185JC, 20190701012GH, 20200801065GH), and the Fundamental Research Funds for the Central Universities (2412019ZD001, 2412020ZD003) 
Author contributions

Z.L., Y.Y. and X.J. contributed equally to this work. Z.L., Y.Y., X.J., Q.S., and S.L. performed the experimental investigations and theoretical calculations. Z.L. and X.B. conceived the concept, designed the project, analyzed the data, and together with G.Z. discussed the results and prepared this manuscript.

Competing interests

The authors declare no competing interests.

Additional information

Supplementary information The online version contains supplementary material available at https://doi.org

Correspondence and requests for materials should be addressed to X.B.

\section{References}

1. Oliveira, K. T., Servilha, B. M., Alves, L. C., Desiderá, A. L. \& Brocksom. T. J. The synthesis of sevenmembered rings in natural products. Stud. Nat. Prod. Chem. 42, 421-463 (2014).

2. Yin, Z., He, Y. \& Chiu, P. Application of $(4+3)$ cycloaddition strategies in the synthesis of natural products. Chem. Soc. Rev. 47, 8881-8924 (2018).

3. Ylijoki, K. E. O. \& Stryker, J. M. [5+2] Cycloaddition reactions in organic and natural product synthesis. Chem. Rev. 113, 2244-2266 (2013).

4. Min, L., Hu, Y.-J., Fan, J., Zhang, W. \& Li, C. Synthetic applications of type II intramolecular cycloadditions. Chem. Soc. Rev. 49, 7015-7043 (2020).

5. Butenschön, H. Seven-membered rings by cyclization at transition metals: [4+3], [3+2+2], [5+2]. Angew. Chem. Int. Ed. 47, 5287-5290 (2008).

6. Trost, B. M., Zuo, Z. \& Schultz, J. E. Transition-metal-catalyzed cycloaddition reactions to access seven-membered rings. Chem. Eur. J. 26, 15354-15377 (2020).

7. Harmata, M. The (4+3)-cycloaddition reaction: simple allylic cations as dienophiles. Chem. Commun. 46, 8886-8903 (2010).

8. Davies, H. M. L. Tandem cyclopropanation / cope rearrangement: a general method for the construction of seven-membered rings. Tetrahedron 49, 5203-5223 (1993).

9. Cheng, Q. Q., Deng, Y., Lankelmaa, M. \& Doyle, M. P. Cycloaddition reactions of enoldiazo compounds. Chem. Soc. Rev. 46, 5425-5443 (2017).

10. The combined $\mathrm{C}-\mathrm{H}$ functionalization / Cope rearrangement: discovery and applications in organic synthesis. Acc. Chem. Res. 45, 923-935 (2012). 
11. Krüger, S. \& Gaich, T. Recent applications of the divinylcyclopropane-cycloheptadiene rearrangement in organic synthesis. Beilstein J. Org. Chem. 10, 163-193 (2014).

12. Davies,H. M. L., Justin R. \& Denton, J. R. Application of donor/acceptor-carbenoids to the synthesis of natural products. Chem. Soc. Rev. 38, 3061-3071 (2009).

13. Miller, L. C., Ndungu, J. M. \& Sarpong, R. Parallel kinetic resolution approach to the cyathane and cyanthiwigin diterpenes using a cyclopropanation / Cope rearrangement. Angew. Chem. Int. Ed. 48, 2398-2402 (2009).

14. Davies, H. M. L., Smith, D. H. \& Korkor, O. Tandem cyclopropanation / Cope rearrangement sequence. Stereospecific [3+4] cycloaddition reaction of vinylcarbenoids with cyclopentadiene. Tetrahedron Lett. 28, 1853-1856 (1987).

15. Davies, H. M. L., Stafford, D. G., Doan, B. D. \& Houser, J. H. Tandem asymmetric cyclopropanation / Cope rearrangement. A highly diastereoselective and enantioselective method for the construction of 1,4-cycloheptadienes. J. Am. Chem. Soc. 120, 3326-3331 (1998).

16. Reddy, R. P. \& Davies, H. M. L. Asymmetric synthesis of tropanes by rhodium-catalyzed [4 + 3] cycloaddition. J. Am. Chem. Soc. 129, 10312-10313 (2007).

17. Guzmán, P. E., Lian, Y. \& Davies, H. M. L. Reversal of the regiochemistry in the rhodium-catalyzed [4+3] cycloaddition between vinyldiazoacetates and dienes. Angew. Chem. Int. Ed. 53, 13083-13087 (2014).

18. Xu, X., Hu, W. H., Zavalij, P. Y. \& Doyle. M. P. Divergent outcomes of carbene transfer reactions from dirhodium and copper-based catalysts separately or in combination. Angew. Chem. Int. Ed. 50, 11152-11155 (2011).

19. Sogo, H. \& Iwasawa, N. Rhenium(I)-catalyzed generation of a, $\beta$-unsaturated carbene complex intermediates from propargyl ethers for the preparation of cycloheptadiene derivatives. Angew. Chem. Int. Ed. 55, 10057-10060 (2016).

20. Shapiro, N. D. \& Toste, F. D. Synthesis of azepines by a gold-catalyzed intermolecular [4 + 3]annulation. J. Am. Chem. Soc. 29, 9244-9245 (2008).

21. Garayalde, D., Krüger, K. \& Nevado, C. Gold-catalyzed cyclopenta- and cycloheptannulation cascades: A stereocontrolled approach to the scaffold of Frondosins A and B. Angew. Chem. Int. Ed. 50, 911915 (2011).

22. Parr, B. T. \& Davies, H. M. L. Rhodium-catalyzed tandem cyclopropanation / Cope rearrangement of 4-alkenyl-1-sulfonyl-1,2,3-triazoles with dienes. Angew. Chem. Int. Ed. 52, 10044-10047 (2013).

23. Shang, H., Wang, Y., Tian, Y., Feng, J. \& Tang, Y. The divergent synthesis of nitrogen heterocycles by rhodium(II)-catalyzed cycloadditions of 1-sulfonyl 1,2,3-triazoles with 1,3-dienes. Angew. Chem. Int. Ed. 53, 5662-5666 (2014).

24. Wang, Y., Cai, P. J. \& Yu, Z. X. Mechanistic study on gold-catalyzed cycloisomerization of dienediynes involving aliphatic $\mathrm{C}-\mathrm{H}$ functionalization and inspiration for developing a new strategy to access polycarbocycles. J. Am. Chem. Soc. 142, 2777-2786 (2020). 
25. Cai, P. J., Wang, Y., Liu, C. H. \& Yu, Z. X. Gold(I)-catalyzed polycyclization of linear dienediynes to seven-membered ring-containing polycycles via tandem cyclopropanation / Cope rearrangement/C$\mathrm{H}$ activation. Org. Lett. 16, 5898-5901 (2014).

26. Kim, S. Y., Park, Y. \& Chung, Y. K. Sequential platinum-catalyzed cycloisomerization and Cope rearrangement of dienynes. Angew. Chem. Int. Ed. 49, 415-418 (2010).

27. Kusama, H., Onizawa, Y. \& Iwasawa, N. W(CO) ${ }_{5}(\mathrm{~L})$-catalyzed tandem intramolecular cyclopropanation / Cope rearrangement for the stereoselective construction of bicyclo[5.3.0]decane framework. J. Am. Chem. Soc. 128, 16500-16501 (2006).

28. Mauleón, P., Zeldin, R. M., González, A. Z. \& Toste, F. D. Ligand-controlled access to [4+2] and [4+3] cycloadditions in gold-catalyzed reactions of allene-dienes. J. Am. Chem. Soc. 131, 6348-6349 (2009).

29. López, F. \& Mascareñas, J. L. [4+2] and [4+3] catalytic cycloadditions of allenes. Chem. Soc. Rev. 43, 2904-2915 (2014).

30. Armengol-Relats, H., Mato, M. \& Echavarren, A. M. Assembly of complex 1,4-cycloheptadienes by $(4+3)$ cycloaddition of rhodium(II) and gold(I) non-acceptor carbenes. Angew. Chem. Int. Ed. 60, 1916-1922 (2021).

31. Galli, C. \& Mandolini, L. The role of ring strain on the ease of ring closure of bifunctional chain molecules. Eur. J. Org. Chem. 2000, 3117-3125 (2000).

32. Davies, H. M. L., Clark, D. M. \& Smith, T. K. [3+4] Cycloaddition reactions of vinyl carbenoids with furans. Tetrahedron Lett. 26, 5659-5662 (1985).

33. Davies, H. M. L., Clark, D. M. Alligood, D. B. \& Eiband, G. R. Mechanistic aspects of formal [3+4] cycloadditions between vinylcarbenoids and furans. Tetrahedron 43, 4265-4270 (1987).

34. Davies, H. M. L., Ahmed, G. \& Churchill, M. R. Asymmetric synthesis of highly functionalized 8oxabicyclo[3.2.1]octene derivatives. J. Am. Chem. Soc. 118, 10774-10782 (1996).

35. Davies, H. M. L., Peng, Z. Q. \& Houser, J. H. Asymmetric synthesis of 1,4-cycloheptadienes and bicyclo[3.2.1] octa-2,6-dienes by rhodium(II) $N$-(p-(tert-butyl) phenylsulfonyl)prolinate catalyzed reactions between vinyldiazomethanes and dienes. Tetrahedron Lett. 35, 8939-8942 (1994).

36. Jackson, K. L., Henderson, J. A., Motoyoshi, H. \& Phillips, A. J. A total synthesis of Norhalichondrin B. Angew. Chem. Int. Ed. 48, 2346-2350 (2009).

37. Xu, J., Caro-Diaz, E. J. E., Batova, A., Sullivan, S. D. E. \& Theodorakis, E. A. Formal synthesis of (-)Englerin A and cytotoxicity studies of truncated englerins. Chem. Asian J. 7, 1052-1060 (2012).

38. Xu, J., Caro-Diaz, E. J. E. \& Theodorakis, E. A. Enantioselective formal synthesis of (-)-Englerin A via a Rh-catalyzed [4+3] cycloaddition reaction. Org. Lett. 12, 3708-3711 (2010).

39. Chow, S., Chow, S., Krainz, T., Bernhardt, P. V. \& Williams, C. M. En route to D-ring inverted Phorbol esters. Org. Lett. 21, 8761-8764 (2019).

40. Hadfielda, M. S. \& Lee, A. L. Gold(I)-catalysed synthesis of conjugated trienes. Chem. Commun. 47, 1333-1335 (2011). 
41. Miki, K., Fujita, M., Uemura, S. \& Ohe, K. Ru-Catalyzed ring-opening and substitution reactions of heteroaromatic compounds using propargylic carboxylates as precursors of vinylcarbenoids. Org. Lett. 8, 1741-1743 (2006).

42. Ikeda, Y., Mura, M., Abo, T., Miki, K. \& Ohe, K. Transition metal-catalyzed ring-opening, substitution, and cyclopropanation reactions via vinylcarbene complexes generated from $O$-propargyl thiocarbamates. Tetrahedron Lett. 48, 6651-6654 (2007).

43. Liu, Z., Cao, S., Yu, W., Wu, J., Yi, F., Anderson, E. A. \& Bi, X. Site-selective C-H benzylation of alkanes with $N$-triftosylhydrazones leading to alkyl aromatics. Chem 6, 2110-211 (2020).

44. Ning, Y., Zhang, X., Gai, Y., Dong, Y., Sivaguru, P., Wang, Y., Reddy, B. R. P., Zanoni, G. \& Bi, X. Difluoroacetaldehyde $N$-triftosylhydrazone (DFHZ-Tfs) as a bench-stable crystalline diazo surrogate. Angew. Chem. Int. Ed. 59, 6473-6481 (2020).

45. Zhang, X., Liu, Z., Yang, X., Dong, Y., Virelli, M., Zanoni, G., Anderson, E. A. \& Bi, X., Use of trifluoroacetaldehyde $N$-tfsylhydrazone as a trifluorodiazoethane surrogate and its synthetic applications. Nat. Commun. 10, 284 (2019).

46. Sivaguru, P. \& Bi, X. Fluoroalkyl $N$-sulfonyl hydrazones: An efficient reagent for the synthesis of fluoroalkylated compounds. Sci. China Chem. 64, 1614-1629 (2021).

47. Doyle, M. P. \& Yan, M. Effective and highly stereoselective coupling with vinyldiazomethanes to form symmetrical trienes. J. Org. Chem. 67, 602-604 (2002).

48. Aggarwal, V. K. et al. A new protocol for the in situ generation of aromatic, heteroaromatic, and unsaturated diazo compounds and its application in catalytic and asymmetric epoxidation of carbonyl compounds. Extensive studies to map out scope and limitations, and rationalization of diastereo- and enantioselectivities. J. Am. Chem. Soc. 125, 10926-10940 (2003).

49. Muñoz-Molina, J. M., Belderrain, T. R. \& Pérez, P. J. Trispyrazolylborate coinage metals complexes: Structural features and catalytic transformations. Coord. Chem. Rev. 390, 171-189 (2019).

50. Caballero, A. \& Pérez, P. J. Catalyst design in the alkane C-H bond functionalization of alkanes by carbene insertion with $\mathrm{Tp}^{\mathrm{x}} \mathrm{M}$ complexes $\left(\mathrm{Tp}^{\mathrm{x}}=\right.$ hydrotrispyrazolylborate ligand, $\left.\mathrm{M}=\mathrm{Cu}, \mathrm{Ag}\right)$. J. Org. Chem. 793, 108-113 (2015).

51. Zhang, X., Liu, Z., Sivaguru, P. \& Bi, X. Silver carbenoids derived from diazo compounds: A historical perspective on challenges and opportunities. Chem Catal. 1, 599-630 (2021).

52. CCDC 2089005 (2) contains the supplementary crystallographic data for this paper. These data can be obtained free of charge from The Cambridge Crystallographic Data Centre via www. ccdc.cam.ac.uk/data_request/cif.

53. Liu, Z., Li, Q., Liao, P. \& Bi, X. Silver-catalyzed [2+1] cyclopropenation of alkynes with unstable diazoalkanes: $N$-nosylhydrazones as room-temperature decomposable diazo surrogates. Chem. Eur.J. 23, 4756-4760 (2017).

54. Liu, Z., Zhang, X., Zanoni, G. \& Bi, X. Silver-catalyzed cyclopropanation of alkenes using Nnosylhydrazones as diazo surrogates. Org. Lett. 19, 6646-6649 (2017). 
55. Thompson, J. L. \& Davies, H. M. L. Enhancement of cyclopropanation chemistry in the silvercatalyzed reactions of aryldiazoacetates. J. Am. Chem. Soc. 129, 6090-6091 (2007).

56. Brewbaker, J. L. \& Hart, H. Cyclization of 3-diazoalkenes to pyrazoles. J. Am. Chem. Soc. 91, 711715 (1969).

57. Yuan, T., Ryckaert, B., Hecke, K. V., Hullaert, J. \& Winne, J. M. Stereoselective gold(I)-catalyzed vinylcyclopropanation via generation of a sulfur-substituted vinyl carbene equivalent. Angew. Chem. Int. Ed. 60, 4070-4074 (2021).

58. Long Min, L., Liu, X. \& Li, C. Total synthesis of natural products with bridged bicyclo[m.n.1] ring systems via type II [5 + 2] cycloaddition. Acc. Chem. Res. 53, 703-718 (2020).

59. Reutrakul, V. \& Kanghae, W. The synthesis of a, $\beta$-unsaturated aldehydes by one-carbon homologation of carbonyl compounds. Tetrahedron Lett. 18, 1377-1380 (1977).

60. Hussain, A., Yousufb, S. K. \& Mukherjee, D. Importance and synthesis of benzannulated mediumsized and macrocyclic rings (BMRs). RSC Adv. 4, 43241-43257 (2014).

61. Davies, H. M. L., McAfee, M. J. \& Oldenburg, C. E. M. Scope and stereochemistry of the tandem intramolecular cyclopropanation / Cope rearrangement sequence. J. Org. Chem. 54, 930-936 (1989).

62. Boger, D. L. \& Brotherton, C. E. Thermal reactions of cyclopropenone ketals. Key mechanistic features and scope of the cycloaddition reactions of delocalized singlet vinylcarbenes: Three-carbon 1,1-/1,3dipoles. J. Am. Chem. Soc. 108, 6695-6713 (1986).

63. Johnson, E. R. et al. Revealing noncovalent interactions. J. Am. Chem. Soc. 132, 6498-6506 (2010).

64. Lu, T. \& Chen, F. Multiwfn: A multifunctional wavefunction analyzer. J. Comput. Chem. 33, 580-592 (2012).

\section{Tables}

Table 1 is in the supplementary files section.

\section{Figures}


a. [4 + 3] Cycloadditions between 1,3-dienes and vinylcarbenoids (extensively studied)

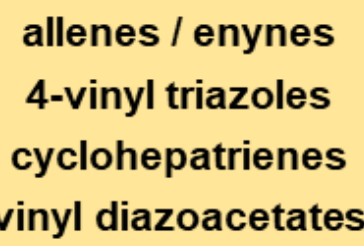

Various vinylcarbene precursors
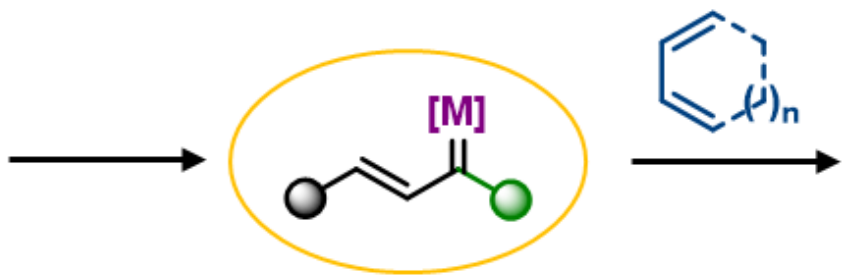

b. [4 + 3] cycloadditions using furans instead of 1,3-dienes (the Davies method)
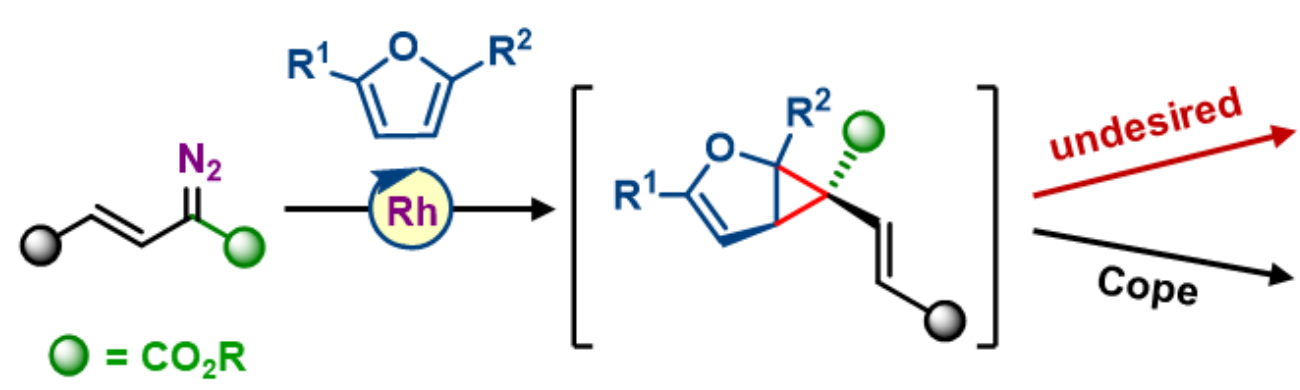<smiles>[R]C(=O)/C=C\C([R])=C(O)/C=C/O</smiles>

Only one kind of carbene precursor

Competitive ring-opening to form trienes

c. Silver-catalyzed [4 + 3] cycloadditions of furans with vinyl- $N$-triftosylhydrazones (this work)<smiles>CN=C(O)C(=O)C=O</smiles>

$+$<smiles>[R]c1cc([R])c([R])o1</smiles>

$\mathrm{O}=\mathrm{H}, \mathrm{CO}_{2} \mathrm{R}, \mathrm{CF}_{3}$
$\mathrm{NaH}, \mathrm{Ag}$ cat.

$$
\text { [4+3] }
$$

cycloaddition

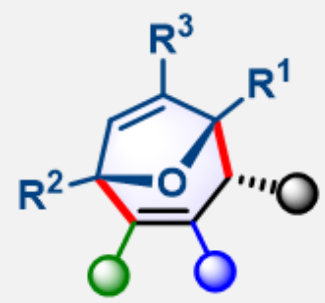<smiles>C[Si](C)(C)S(=O)(=O)c1ccccc1C(F)(F)F</smiles>

Triftosyl (Tfs)

\section{Broad substrate scope}

\section{Regio- and stereoselectivity}

Figure 1

Assembly of seven-membered carbocycles by [ $4+3]$ cycloaddition of 1,3-dienes/furans with vinylcarbenes. 


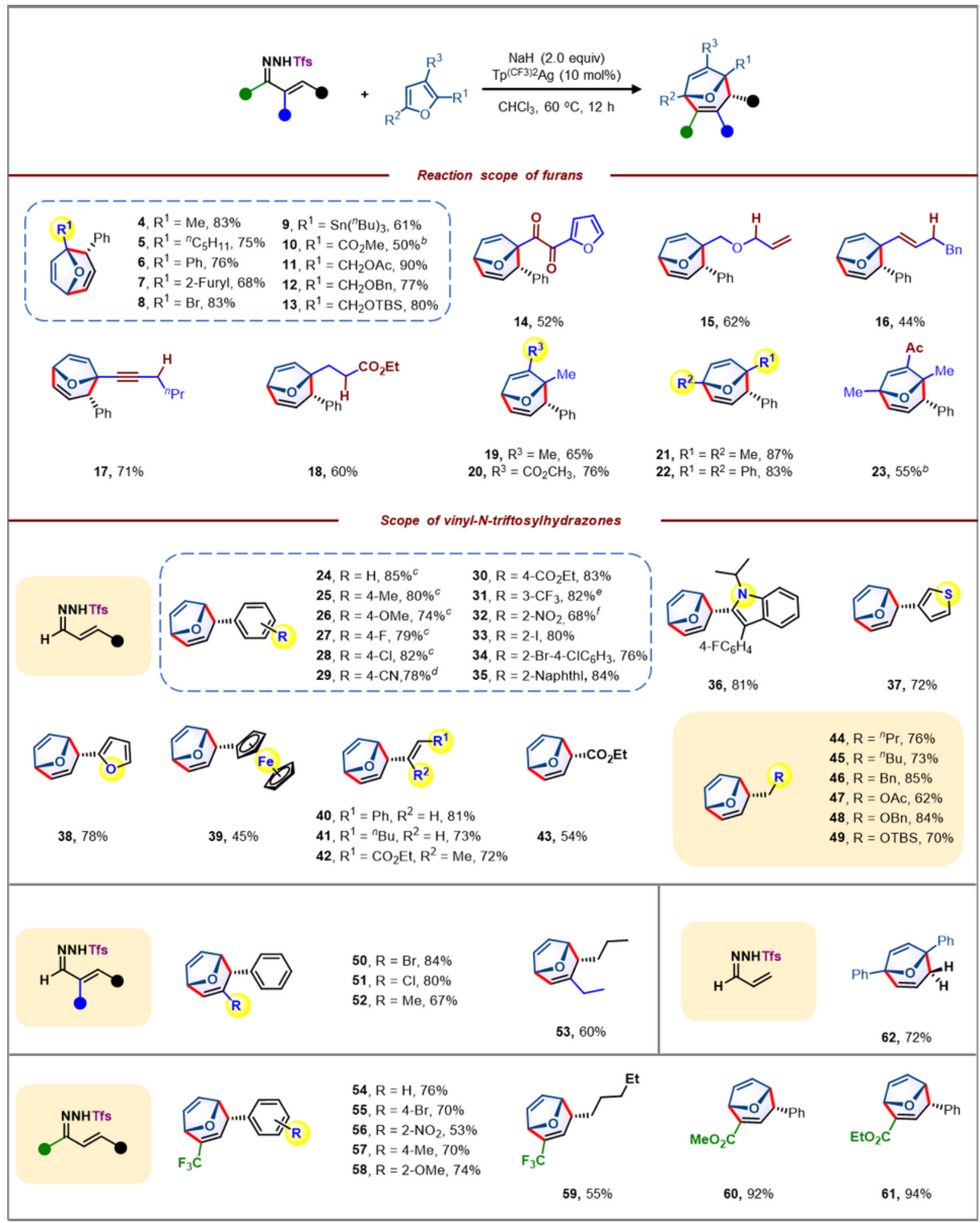

Figure 2

Substrate scope for intramolecular [4 + 3] cycloaddition. ${ }^{a}$ Reaction conditions: Vinyl- $N$-triftosylhydrazone (0.3 mmol), furan $(0.6 \mathrm{mmol}), \mathrm{NaH}(0.6 \mathrm{mmol}),, \mathrm{Tp}^{(\mathrm{CF} 3) 2} \mathrm{Ag}(10 \mathrm{~mol} \%)$ in $\mathrm{CHCl}_{3}(3.0 \mathrm{~mL})$ was stirred at 60 ${ }^{\circ} \mathrm{C}$ for $12 \mathrm{~h}$ under argon atmosphere. Isolated yield. ${ }^{\mathrm{b}} 20 \mathrm{~mol} \% \mathrm{Tp}(\mathrm{CF} 3) 2 \mathrm{Ag}$ in $\mathrm{CHCl}_{3}$ at $60{ }^{\circ} \mathrm{C}$ for $24 \mathrm{~h}$. ${ }^{\mathrm{c}} 5 \mathrm{~h}$. $\mathrm{d}_{24} \mathrm{~h}$. eat $80{ }^{\circ} \mathrm{C}$ for $24 \mathrm{~h} .{ }^{\mathrm{f}}$ in $\mathrm{PhCF}_{3}$ at $60^{\circ} \mathrm{C}$ for $24 \mathrm{~h}$. 


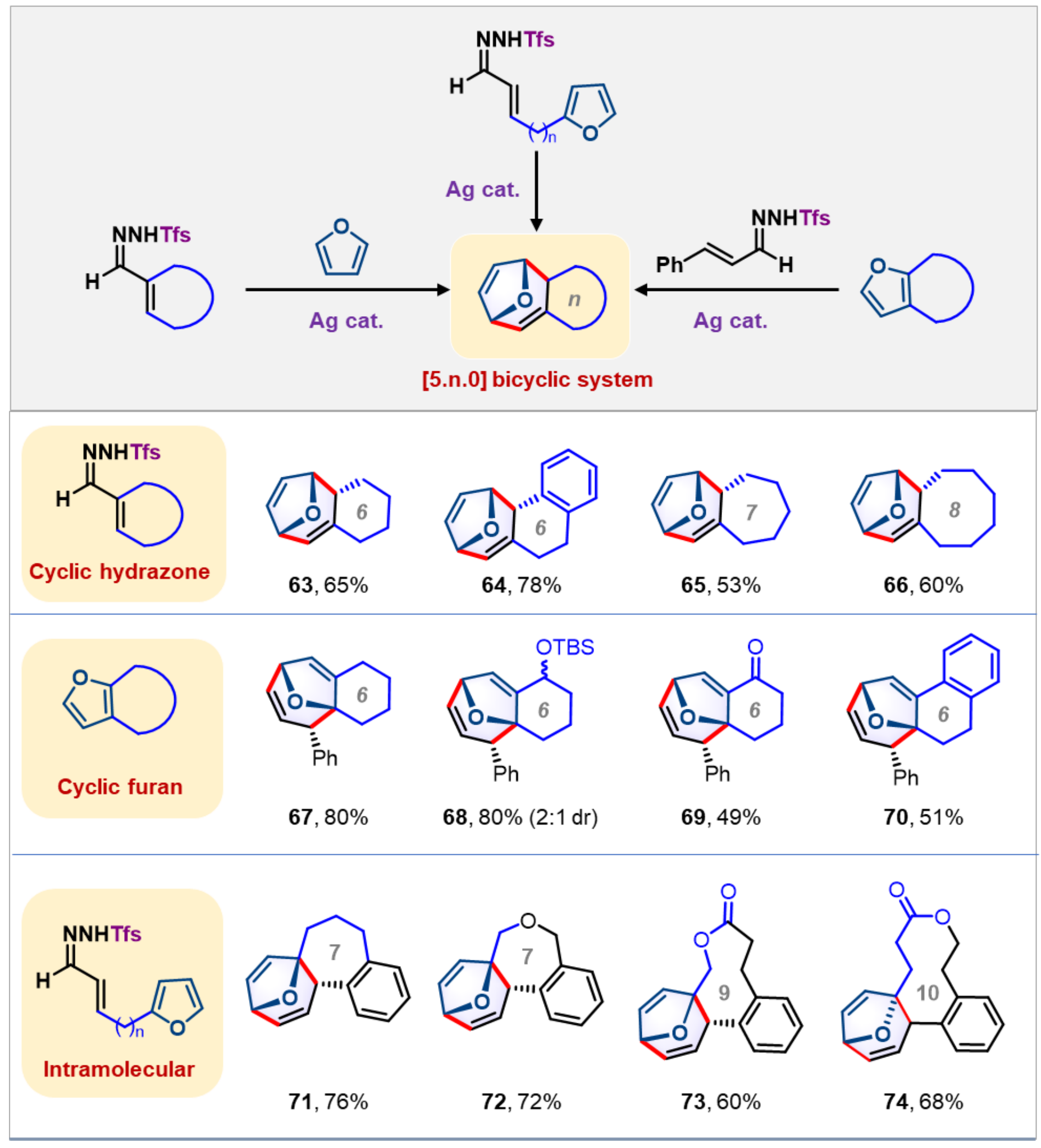

Figure 3

Collective synthesis of oxa-bridged bicyclo[5.n.0] ring systems. 


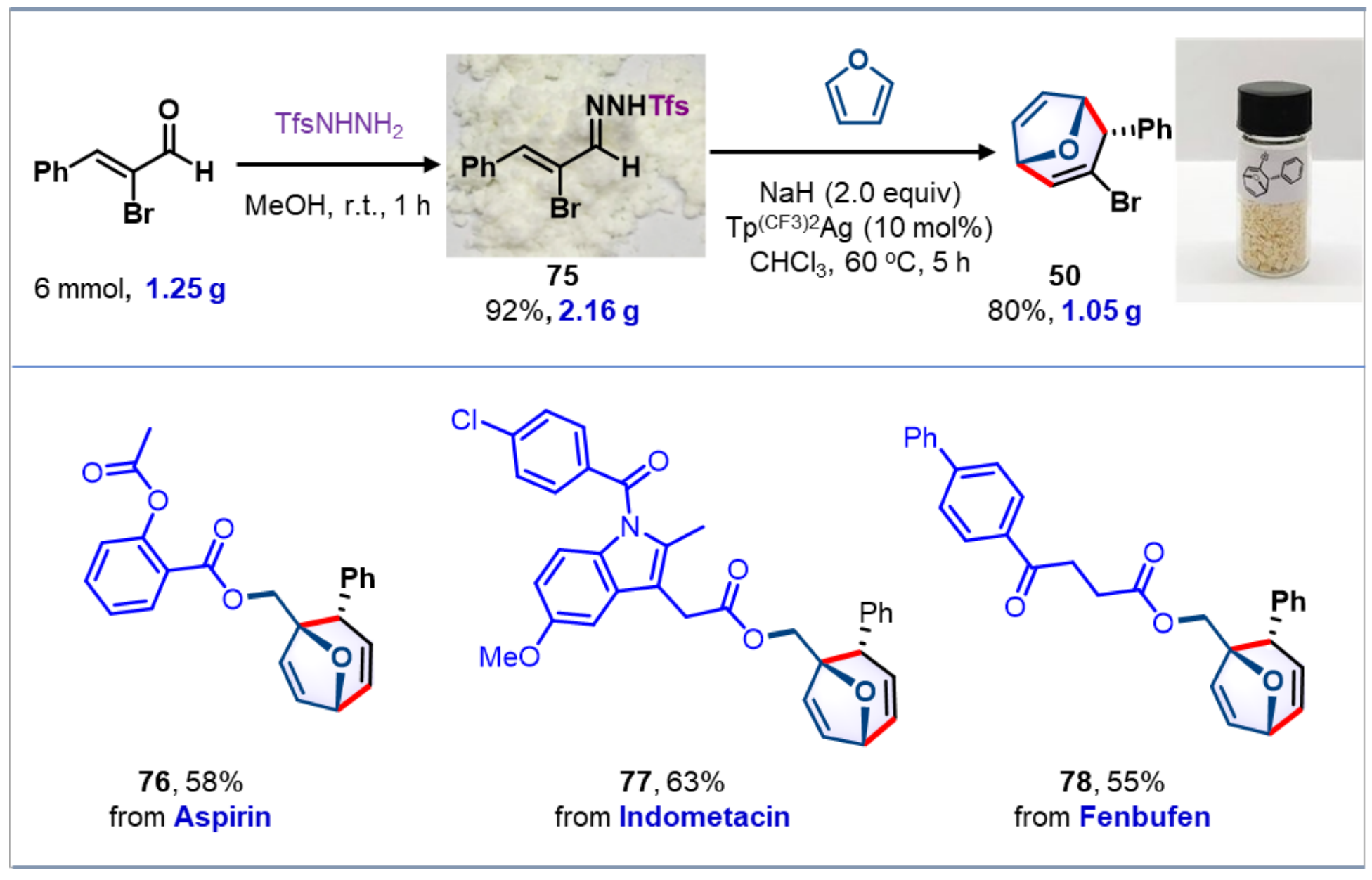

Figure 4

Gram-scale reaction and late-stage modifications. 
(a) Control experiments

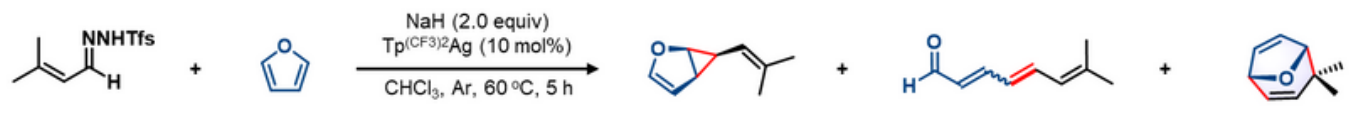

79

$80,57 \%$

$81,12 \%$ NMR yield

82 , trace

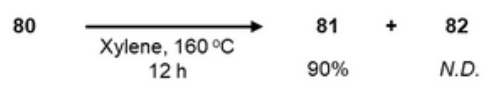

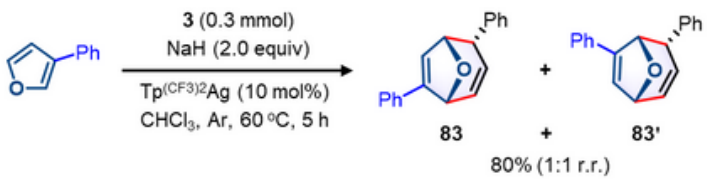

(b) DFT calculations: plausible reaction pathways

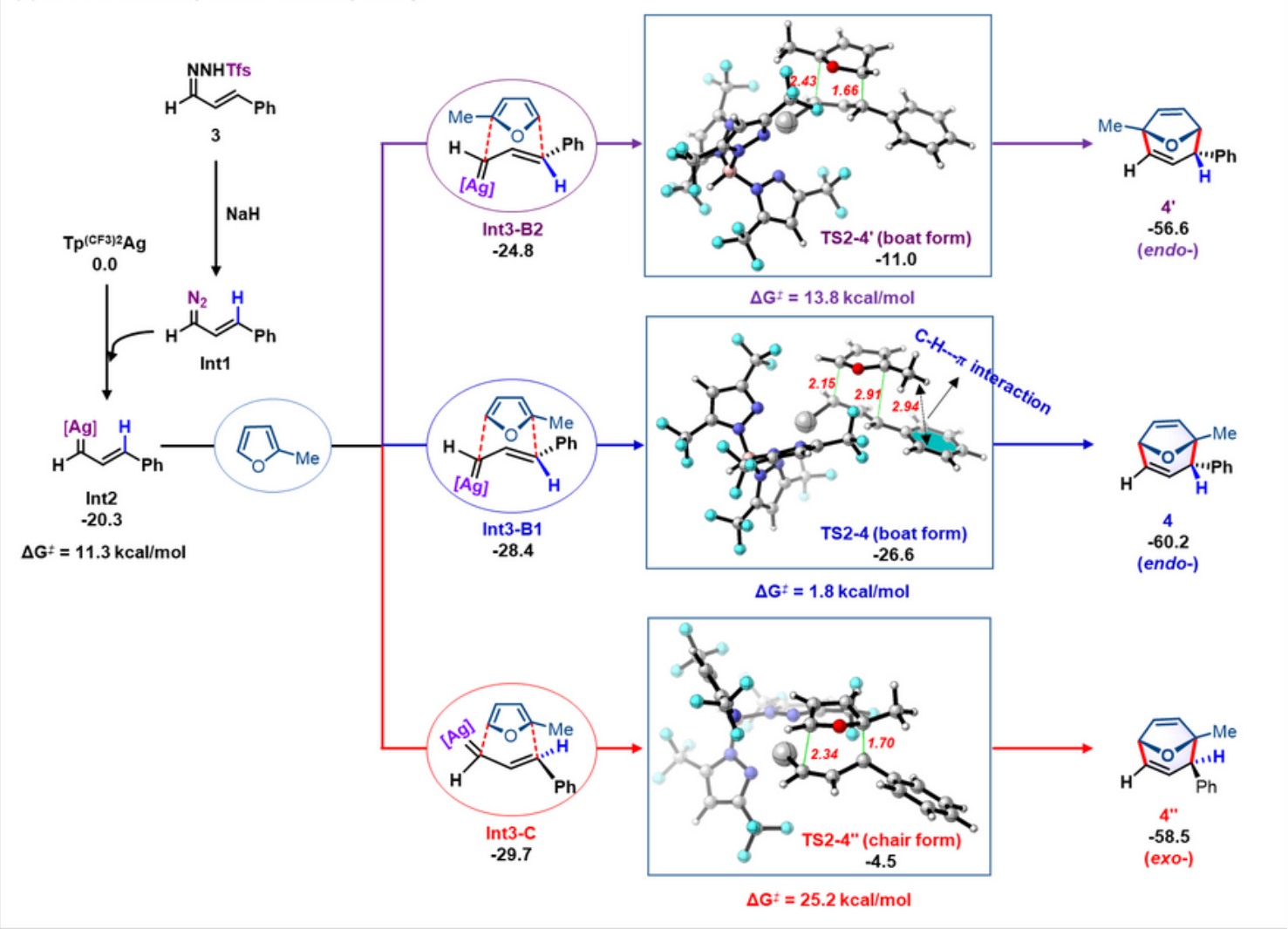

(c) $\mathrm{NCl}$ analysis of transition states

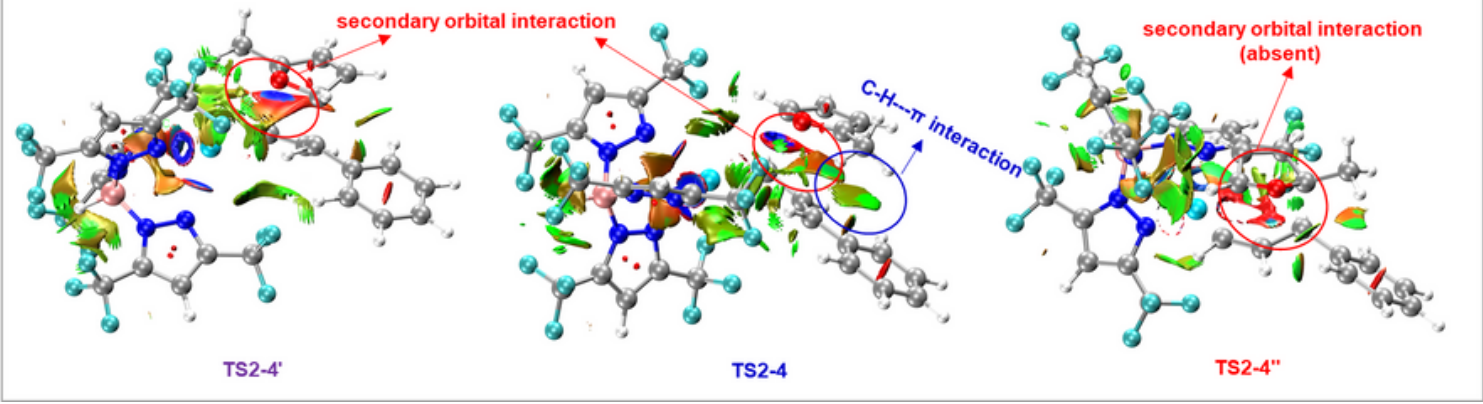

Figure 5

Mechanistic investigations. (a) Control experiments. (b) A concerted [4 + 3] cycloaddition was proposed by computational studies at the B3LYP-D3(BJ)//6-31G(d,p)-SDD(Ag) level of theory. The relative Gibbs free energies are given in $\mathrm{kcal} / \mathrm{mol}$ (black); the distances are given in angstroms (red). (c) $\mathrm{NCl}$ analysis of transition states for [4 + 3] cycloadditions. Blue: attraction; green: weak interaction; red: steric effect. 


\section{Supplementary Files}

This is a list of supplementary files associated with this preprint. Click to download.

- Table1.docx

- Supplementarylnformation.docx 